\title{
EFFECTS OF STRUCTURAL OPTIMIZATION ON PRACTICAL ROOF TRUSS CONSTRUCTION
}

UDC: 624.041

Original scientific paper

https://doi.org/10.18485/aeletters.2020.5.2.1

\author{
Nenad Petrović ${ }^{1}{ }^{*}$, Nenad Kostić ${ }^{1}$, Nenad Marjanović ${ }^{1}$, Jelena Živković ${ }^{1}$, loana I Cofaru ${ }^{2}$ \\ ${ }^{1}$ University of Kragujevac, Faculty of Engineering, Serbia \\ ${ }^{2}$ University "Lucian Blaga" of Sibiu Department of Industrial Machines and Equipment, Romania
}

\begin{abstract}
:
In truss structural optimization the most frequently optimized factor of a structure is its weight. The minimization of weight contributes not only to savings in material, but also in other aspects of the structure such as number of elements used, number of welds needed, outer surface area, etc. This research aims to show the difference in optimal solutions for four different topological cases of a typical trapezoidal roof truss looking at their effects on overall outer surface area. The truss layouts are optimized for sizing, and a combination of sizing and shape with a minimal weight objective function. In order to ensure the most practically applicable solutions the example optimized in this paper uses dynamic constraints for buckling, stress constraints, and nodal displacement constraints. The overall outer surface area for all cases is compared, as surface protection accounts for a substantial part of the total cost of roof truss construction. Optimal solutions show a lack of correlation between weight and surface area, which is discussed in the conclusion.
\end{abstract}

\section{ARTICLE HISTORY}

Received: 13.05.2020.

Accepted: 02.06.2020.

Available: 30.06.2020.

\section{KEYWORDS}

Roof truss, structural optimization, Euler buckling, dynamic constraints, genetic algorithm

\section{INTRODUCTION}

In the search for more economical design solutions engineers today rely on optimization processes to find concepts which might not otherwise be logical or would require numerous iterations of analytical calculations. For practical applications complexity of the optimization process is increased significantly with multiple aspects of the construction being optimized and with every constraint which is added to avoid unusable solutions. The minimal weight optimization is generally approached through optimizing either sizing (varying the cross sections of bars or bar groups), shape (varying the positions of nodes), topology (adding or removing elements between nodes), or one of their combinations. The constraints limiting the search-space maximal allowed stress and maximal allowed displacement are generally found in literature. In order to maintain construction stability dynamic constraints for buckling must also be implemented.

Tejani et al. [1] conducted simultaneous sizing, shape and topology optimization of planar and space trusses without considering buckling. Their approach did however account for possible unacceptable topologies using Grubler's criterion. Researchers in [2] compared sizing, topology and shape optimization results of planar and space trusses to sequential optimization of these three criteria as well as a simultaneous optimization of all three. Results showed great improvements in results of simultaneous optimization compared to initial models and single aspect optimization, however these examples did not include Euler buckling constraints. Gonçalves et al. [3] have used discrete sizing variables with buckling constraints on 10,37 , and 20 bar truss examples in a few combinations of optimization types with great results. Authors in [4-9] included dynamic 
constraints for buckling in their research using various optimization methods in order to optimize different aspects of examples. Researchers in [10] presented the need for using buckling constraints on sizing optimization examples of planar and space trusses. Authors in [11] conducted global buckling and frequency analyses on $2 \mathrm{D}$ and $3 \mathrm{D}$ trusses. In $[12,13]$ showed the influence of using discrete cross-section variables in optimization, which provide useable results. The complexity of these problems however require optimization methods which can operate with a small number of known inputs and navigate the vast searchspace.

Roof truss structures are a particularly interesting area of research when it comes to truss optimization, as there is a lot of possibility for practical application. Reda et al. [14] investigated the overall behaviour of cold formed steel roof trusses using finite element analysis and to predict the failure location in the truss assembly and its cause. This was done by calculating the demand to capacity ratios using Direct Strength Method (DSM) for each component of the truss. Dawe et al. [15] studied various practical strengthening techniques in order to achieve a desired behaviour and an increased capacity using both the conventional interaction design equations and the direct strength method. Research of this nature drives forward the development of new techniques in truss optimization and design.

In this paper a typical roof truss was optimized for sizing, shape and their simultaneous combination. An original software was developed for the purposes of using genetic algorithm optimization which includes stress, displacement and buckling constraints with discrete crosssectional variables and calculates the total outer surface area of the optimal truss. As surface protections plays a big part in the overall cost of constructing a roof truss, up to a quarter of the total cost in fact, this aspect of the optimal design concepts is compared to show whether a minimal weight design provides savings in other aspects of the construction as well.

\section{TRUSS STRUCTURAL OPTIMIZATION}

Truss structural optimization implies the simultaneous optimization of sizing, shape, and topology. This is not always possible depending on the initial design of the truss being optimized and practical conditions of the location and loading of the truss. It is most common to see the optimization of one or a combination of two of these aspects of a structure. In order to create a practically applicable optimal model it is necessary to create realistic loading cases and supports in the initial model. The variables for sizing must be a discrete set which should reflect available crosssection profiles typically used in such structures and have the bars to which they can be assigned grouped in order to ensure the possibility and ease of assembly. When optimizing the shape spatial constraints must be considered to ensure that no nodes (joints) are obstructing their intended surroundings or clash with bars and joints. Topological optimization depends on the structural stability of the construction. Removal of bars in this optimization aspect must ensure that the truss does not become a mechanism. This is why topological optimization is not always possible. The most common objective function of truss structural optimization is the minimization of weight. The minimum weight design problem is defined in expression (1) which also gives the basic constraints used in truss structural optimization.

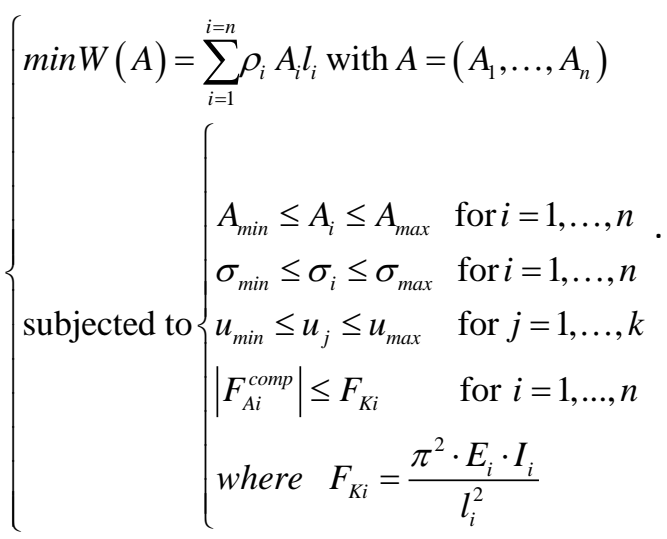

In expression (1) is the number of truss elements, $k$ is the number of nodes, $l_{i}$ is the length of the $i^{\text {th }}$ element, $A_{i}$ is the area of the $i^{\text {th }}$ element's cross-section profile, $\sigma_{i}$ is the stress of the $i^{\text {th }}$ element, $\mathrm{u}_{\mathrm{j}}$ is displacement of the $\mathrm{j}^{\text {th }}$ node. $\mathrm{F}_{\mathrm{Ai}}$ comp is the axial compression force, $F_{K i}$ is Euler's critical load, $E_{i}$ is the modulus of elasticity, and $I_{i}$ is the minimum area moment of inertia of the crosssection profile of the of the $i_{\text {th }}$ element.

Each addition of a constraint increases the complexity of the problem and makes the finding of global optima more difficult. By adding dynamic constraints such as Euler buckling this becomes an even bigger problem. These constraints are considered to be dynamic as they change with each iteration of optimization since the crosssection profile changes with it the moment of 
inertia, and the change of shape changes the length of elements. Topological changes as well as are given in Table 1. These profiles were selected as they are the most commonly used and

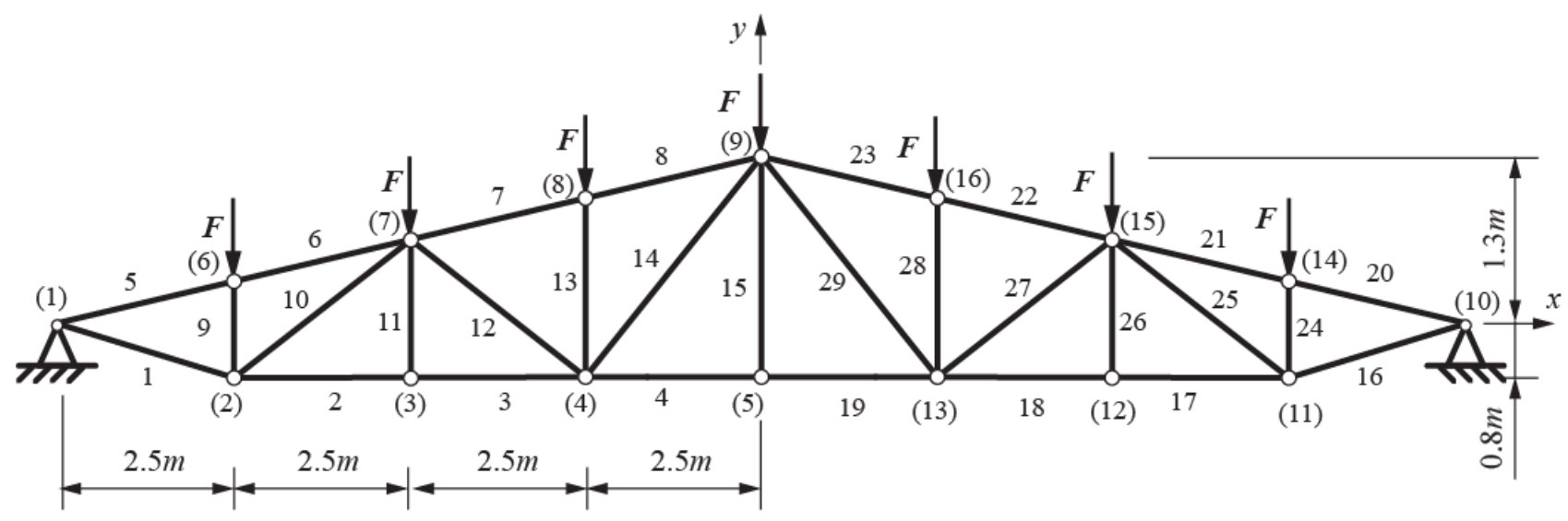

Fig.1. Layout of trapezoidal roof truss problem

shape changes can also in some cases influence the direction of forces in bars, so the constraint would apply do different bars in different iterations of optimization.

These types of problems require non-linear optimization algorithms which can handle large numbers of variables. Heuristic methods are generally used in engineering practice to solve these types of problems as they have favourable characteristics such as: the ability to work with a large number of variables, overcoming local extremes, efficiency of operation, speed, low threshold for needed facts about the problem in order to find a solution, etc.

\section{EXAMPLE}

The roof truss problem used for this example is a typically used layout for roof spans of under $36 \mathrm{~m}$ in length. The specific example used in this paper and its layout of nodes and bars is given in Fig.1. The first case configuration uses 16 nodes and is the basis for other topological cases. For this example S235JRG2 steel is used with a Young modulus of 210 000MPa and a density of $7.85 \mathrm{~kg} / \mathrm{m}^{3}$. The structure is subjected to point loads of $\mathrm{F}=17.652 \mathrm{kN}$ in the nodes shown in Fig.1. which represent a maximal load from snow.

Constraints for the problem are a compression and tension strass limit of $180 \mathrm{MPa}$ for all bars, a maximal allowed displacement of $\pm 0.036 \mathrm{~m}$ for all nodes, and dynamic constraints for Euler buckling for all bars in every iteration

Moments of inertia for the 40 possible profiles were taken from various vendor's catalogues and commercially available in Serbia.

Shape optimization constraints for node coordinates are grouped according to symmetry around the $\mathrm{y}$ axis and are given, in meters, as follows:

$$
\begin{aligned}
& 5,5 \leq-x_{2}=x_{11} \leq 8.5 \\
& 3 \leq-x_{3}=x_{12} \leq 7 \\
& 0.5 \leq-x_{4}=x_{13} \leq 4.5 \\
& 0 \leq y_{2-5}=y_{11-13} \leq-0.8
\end{aligned}
$$

Cross-section profiles of elements are grouped into 9 different groups and are symmetrically arranged. The cross-section profile groups are as follows: $A_{(1-4)}=A_{(16-19)}, A_{(5-8)}=A_{(20-23)}, A_{(9)}=A_{(24)}, A_{(10)}$ $=A_{(25)}, A_{(11)}=A_{(26)}, A_{(12)}=A_{(27)}, A_{(13)}=A_{(28)}, A_{(14)}=$ $A_{(29),} A_{(15)}$. These groups are made to avoid solutions with changes in cross-section along a line of elements which are in arranged in series which would require additional critical load parameters. Only square HSS profiles were used in the list of discrete sizing variables for cross-section profiles according to table.

Topology optimization for this example was not used as the removal of vertical elements would create a mechanism. In order to have various topological layouts for this example four possible layouts were used (Fig.2-5). In each case where a vertical member is removed from the layout, the two vertical beams which connected to it were replaced with a single beam and the node was omitted. 
Table 1. HSS profiles used for the example and their moments of inertia

\begin{tabular}{|c|c|c|c|c|}
\hline \multirow{2}{*}{$\begin{array}{c}\text { Profile } \\
\text { dimensions } \\
{[\mathrm{mm}]}\end{array}$} & \multicolumn{4}{|c|}{$\begin{array}{c}\text { Moments of inertia }\left[\mathrm{cm}^{4}\right] \text { according to wall } \\
\text { thickness of profile }[\mathrm{mm}]\end{array}$} \\
\hline & 3 & 4 & 5 & 6 \\
\hline $40 \times 40$ & 8.6 & 11.1 & - & - \\
\hline $45 \times 45$ & 14.4 & 17.6 & - & - \\
\hline $50 \times 50$ & 19.5 & 23.7 & 27 & - \\
\hline $60 \times 60$ & 35.1 & 43.6 & 50.5 & - \\
\hline $70 \times 70$ & 56.1 & 68.9 & - & - \\
\hline $80 \times 80$ & 87.8 & 111 & 131 & 149 \\
\hline $90 \times 90$ & 127 & 162 & 193 & 220 \\
\hline $100 \times 100$ & 177 & 226 & 271 & 311 \\
\hline $110 \times 110$ & 235.9 & 300.3 & 357.4 & 439.8 \\
\hline $120 \times 120$ & 317.2 & 409.5 & 498.6 & 562 \\
\hline $130 \times 130$ & 397.3 & 510.2 & 612.8 & 748.3 \\
\hline $140 \times 140$ & 510 & 661.5 & 805.8 & 945.8 \\
\hline
\end{tabular}

All layout cases were optimized for sizing and a simultaneous combination of sizing and shape using an original software developed by the authors.

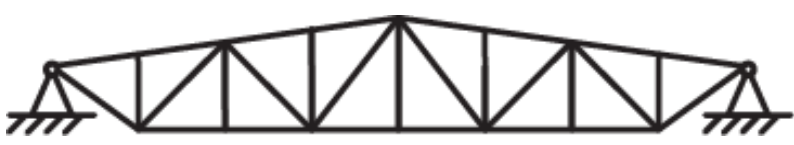

Fig.2. Bar layout of topological case 1

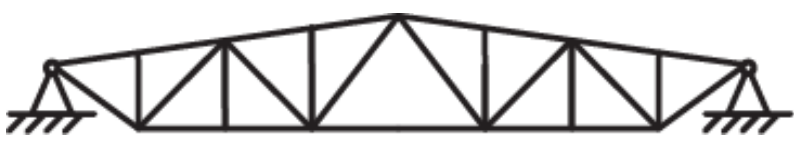

Fig.3. Bar layout of topological case 2

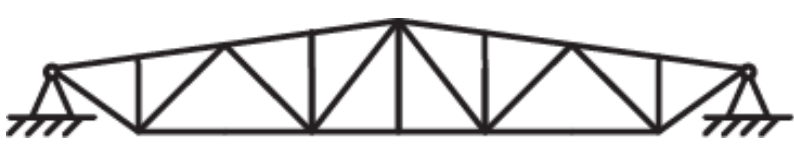

Fig.4. Bar layout of topological case 3

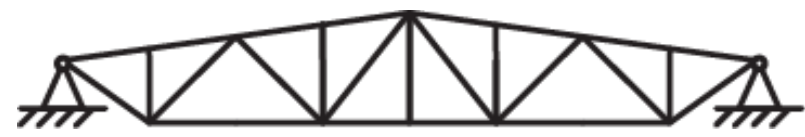

Fig.5. Bar layout of topological case 4

The optimization method used was genetic algorithm due to its availability and favourable characteristics.

\section{RESULTS}

A small number of shape variables does not allow for drastic differences in results when optimizing for shape in this example. Table 2 gives optimal node coordinates according to case for simultaneous optimization of sizing and shape. Since node 5 has the same y coordinate as nodes 2-4 and 11-13, yet does not exist in cases 2 and 4 so it is not given in the table.

Table 2. Coordinates of nodes for optimal sizing and shape solutions according to case

\begin{tabular}{|c|c|c|c|c|}
\hline $\begin{array}{c}\text { Node } \\
\text { coordinates }\end{array}$ & Case 1 & Case 2 & Case 3 & Case 4 \\
\hline$-x_{2}, x_{11}$ & 7.12 & 7.01 & 7.02 & 7.27 \\
\hline$-x_{3}, x_{12}$ & 6.06 & 5.00 & - & - \\
\hline$-x_{4}, x_{13}$ & 2.66 & 2.65 & 2.48 & 2.58 \\
\hline$y_{2-4}, y_{11-13}$ & 0.80 & 0.80 & 0.8 & 0.8 \\
\hline
\end{tabular}

Fig.6 shows the differences in optimal weight for all four topological layout cases for both sizing and sizing shape combination.

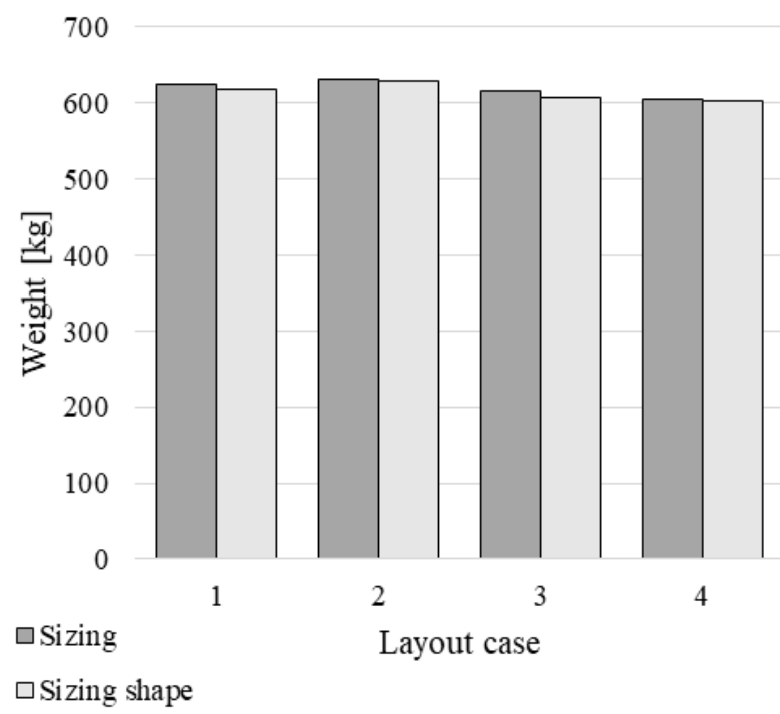

Fig.6. Difference in optimal weight according to topological layout case and optimization type

Table 3 shows the optimal cross-section profiles according to bar group, weight, and outer area for all four topological cases according to the aspect of the truss being optimized.

Overall outer surface areas of optimal model's bars are shown in Fig.7 for all cases and optimization aspects. 
Table 3. Optimal cross section profile, weight and outer area results for all four topological cases

\begin{tabular}{|c|c|c|c|c|c|c|c|c|}
\hline \multirow{3}{*}{$\begin{array}{c}\text { Profile } \\
\text { dimensions of } \\
\text { element group } \\
\text { [mm] }\end{array}$} & \multirow{2}{*}{\multicolumn{2}{|c|}{ Case 1}} & \multirow{2}{*}{\multicolumn{2}{|c|}{ Case 2}} & \multirow{2}{*}{\multicolumn{2}{|c|}{ Case 3}} & \multirow{2}{*}{\multicolumn{2}{|c|}{ Case 4}} \\
\hline & & & & & & & & \\
\hline & Sizing & Sizing shape & Sizing & $\begin{array}{l}\text { Sizing } \\
\text { shape }\end{array}$ & Sizing & $\begin{array}{l}\text { Sizing } \\
\text { shape }\end{array}$ & Sizing & Sizing shape \\
\hline$A_{(1-4,16-19)}$ & $90 \times 90 \times 5$ & $140 \times 140 \times 3$ & $90 \times 90 \times 4$ & $90 \times 90 \times 5$ & $90 \times 90 \times 5$ & $90 \times 90 \times 5$ & 140x140x3 & $140 \times 140 \times 3$ \\
\hline$A_{(5-8,20-23)}$ & $90 \times 90 \times 5$ & $140 \times 140 \times 3$ & $90 \times 90 \times 6$ & $90 \times 90 \times 5$ & $90 \times 90 \times 5$ & $90 \times 90 \times 5$ & $140 \times 140 \times 3$ & $140 \times 140 \times 3$ \\
\hline$A_{(9,24)}$ & $40 \times 40 \times 3$ & $45 \times 45 \times 3$ & $50 \times 50 \times 3$ & $50 \times 50 \times 3$ & $40 \times 40 \times 3$ & $40 \times 40 \times 3$ & $40 \times 40 \times 3$ & $40 \times 40 \times 3$ \\
\hline$A_{(10,25)}$ & $60 \times 60 \times 3$ & $45 \times 45 \times 3$ & $50 \times 50 \times 3$ & $50 \times 50 \times 3$ & $60 \times 60 \times 3$ & $50 \times 50 \times 3$ & $60 \times 60 \times 3$ & $60 \times 60 \times 3$ \\
\hline$A_{(11,26)}$ & $40 \times 40 \times 3$ & $40 \times 40 \times 3$ & $50 \times 50 \times 3$ & $50 \times 50 \times 3$ & - & - & - & - \\
\hline$A_{(12,27)}$ & $40 \times 40 \times 3$ & $45 \times 45 \times 3$ & $50 \times 50 \times 3$ & $50 \times 50 \times 3$ & $40 \times 40 \times 3$ & $40 \times 40 \times 3$ & $40 \times 40 \times 3$ & $40 \times 40 \times 3$ \\
\hline$A_{(13,28)}$ & $40 \times 40 \times 3$ & $45 \times 45 \times 3$ & $50 \times 50 \times 3$ & $50 \times 50 \times 3$ & $40 \times 40 \times 3$ & $40 \times 40 \times 3$ & $40 \times 40 \times 3$ & $40 \times 40 \times 3$ \\
\hline$A_{(14,29)}$ & $40 \times 40 \times 3$ & $40 \times 40 \times 3$ & $50 \times 50 \times 3$ & $50 \times 50 \times 3$ & $40 \times 40 \times 3$ & $40 \times 40 \times 3$ & $40 \times 40 \times 3$ & $40 \times 40 \times 3$ \\
\hline$A_{(15)}$ & $40 \times 40 \times 3$ & $40 \times 40 \times 3$ & - & - & $40 \times 40 \times 3$ & $40 \times 40 \times 3$ & - & - \\
\hline Weight [kg] & 625.28 & 617.637 & 630.415 & 629.502 & 615.7 & 606.993 & 604.068 & 601.89 \\
\hline Area $\left[\mathrm{m}^{2}\right]$ & 19.635 & 27.596 & 19.909 & 19.768 & 19.171 & 18.797 & 26.919 & 26.819 \\
\hline
\end{tabular}

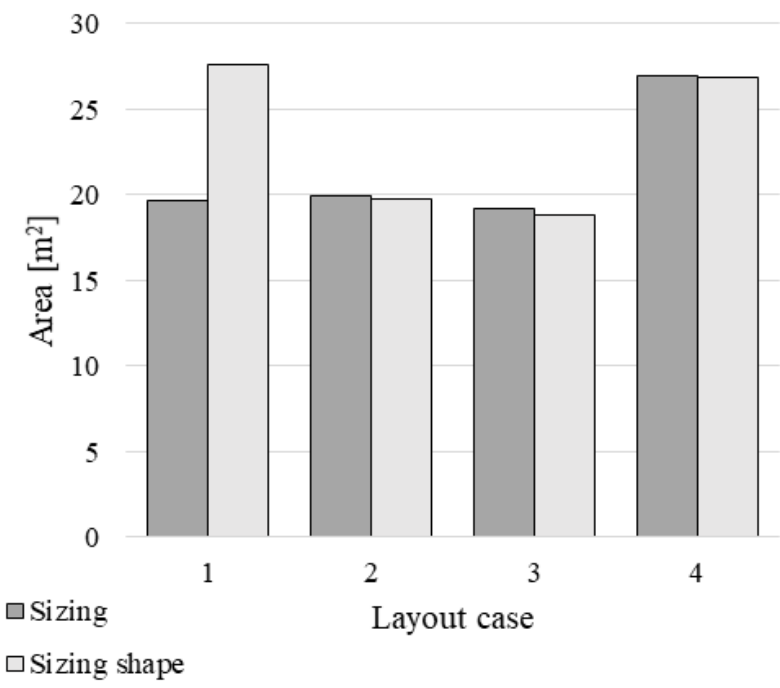

Fig.7. Difference in overall outer surface area according to topological layout case and optimization type

\section{CONCLUSION}

Truss structural optimization is a complex problem which requires an in-depth understanding of truss design problems. When optimizing for minimal weight the general goal is to decrease the amount of material used in order to decrease overall costs. This approach, however, can result in increases of other facets of the truss construction. As all roof trusses must be painted upon assembly there is always the cost of surface protection to consider. The amount of paint or other coating required is dependent on the total outer surface area of the bars used. This research is focused on the influence of optimizing various aspects of a practical construction and the influence the decreased weight has on the overall surface area of the truss and thereby the overall final cost.

The example used in this research is a typical roof truss for a $20 \mathrm{~m}$ wide roof. Since typical topological optimization would cause mechanical instability four topological layout cases were optimized for sizing and a combination of sizing and shape. The analytically calculated solution for this truss using $100 \times 100 \times 4$ in the lower part, $90 \times 90 \times 6$ in the top part, and $50 \times 50 \times 4$ in the infill of the initial (case 1) model has a weight of $699.683 \mathrm{~kg}$, and an outer surface area of $21.139 \mathrm{~m}$. When compared to optimized weights case 1 gives a $10.6 \%$ lighter truss for sizing and $11.8 \%$ lighter truss for sizing and shape optimization. Case 2 is lighter by $9.9 \%$ and $10.0 \%$, case 3 by $12.0 \%$ and $13.2 \%$, and case 4 by $13.7 \%$ and $14.0 \%$, for sizing and shape respectfully.

The decrease in weight does not correlate with the decrease in surface area. Namely layout case 1 has a $7.1 \%$ smaller overall surface area than the analytically calculated model for sizing optimization, while for sizing and shape the area is in fact larger by $30.5 \%$. Layout case 2 has a smaller overall surface for both sizing and sizing and shape optimization, $5.8 \%$, and $6.5 \%$ respectively. Layout case 3 also has a smaller surface in both cases with a $9.3 \%$ decrease for sizing and $11.07 \%$ decrease for sizing and shape optimized models. Case 4, even though it has the fewest elements of all the cases and the lowest weights for both sizing and shape optimization the overall surface areas are $27.3 \%$ 
and $26.9 \%$ larger than the analytically calculated model's respectfully.

The difference in overall surface area cannot be attributed to large changes in element lengths as the nodes in all cases where shape optimization is considered do not differ drastically from the initial setup. The main reason for an especially large difference between other cases and case 4 optimal models' overall surface areas is largely due to the profiles having smaller wall thicknesses and larger outer dimensions of profiles used. This is logical when considering that the ideal cross section profile is with the thinnest possible walls with material placed furthest from the central axes.

In conclusion, it can be noted that the minimal weight design for trusses using a combined approach of sizing, shape and topology optimization gives the best results in terms of weight, it might not be the case when looking at the effects it has on other aspects of a construction. It is the opinion of the authors that more research needs to be done in terms of the possibility of multi criteria optimization where when considering costs, all influential aspects can be considered in order to achieve the best possible results.

\section{REFERENCES}

[1] G.G. Tejani, V.J. Savsani, V.K. Patel, P.V. Savsani, Size, shape, and topology optimization of planar and space trusses using mutation-based improved metaheuristics. Journal of Computational Design and Engineering, 5 (2), 2018: 198-214.

https://doi.org/10.1016/i.jcde.2017.10.001

[2] T.E. Müller, E.v.d. Klashorst, A Quantitative Comparison Between Size, Shape, Topology and Simultaneous Optimization for Truss Structures. Latin American Journal of Solids and Structures, 14 (12), 2017: 2221-2242.

http://dx.doi.org/10.1590/1679-78253900

[3] M.S. Gonçalves, R.H. Lopez, L.F.F. Miguel, Search group algorithm: A new metaheuristic method for the optimization of truss structures. Computers \& Structures, 153, 2015: 165-184.

https://doi.org/10.1016/j.compstruc.2015.03.003

[4] H. Assimi, A. Jamali, A hybrid algorithm coupling genetic programming and NelderMead for topology and size optimization of trusses with static and dynamic constraints. Expert Systems with Applications, 95, 2018: 127-141.

https://doi.org/10.1016/i.eswa.2017.11.035
[5] E. Grande, M. Imbimbo, V. Tomei, Role of global buckling in the optimization process of grid shells: Design strategies. Engineering Structures, 156, 2018: 260-270.

https://doi.org/10.1016/i.engstruct.2017.11.049

[6] H. Madah, O. Amir, Truss optimization with buckling considerations using geometrically nonlinear beam modelling. Computers \& Structures, 192, 2017: 233-247.

https://doi.org/10.1016/j.compstruc.2017.07.023

[7] A. Xiao, B. Wang, C. Sun, S. Zhang, Z. Yang, Fitness Estimation Based Particle Swarm Optimization Algorithm for Layout Design of Truss Structures. Mathematical Problems in Engineering, 2014, 2014: 1-11.

https://doi.org/10.1155/2014/671872

[8] A. Ahrari, A.A. Atai, Fully Stressed Design Evolution Strategy for Shape and Size Optimization of Truss Structures. Computers \& Structures, 123, 2013: 58-67.

https://doi.org/10.1016/i.compstruc.2013.04.013

[9] G. Janevski, M. Stamenković, M. Seabra, The Critical Load Parameter of a Timoshenko Beam with One-Step Change in Cross Section. Facta Universitatis, Series: Mechanical Engineering, 12 (3), 2014: 261-276.

[10] N. Petrovic, N. Marjanovic, N. Kostic, M. Blagojevic, M. Matejic, S. Troha, Effects of introducing dynamic constraints for buckling to truss sizing optimization problems. FME Transaction, 46 (1), 2018: 117-123.

https://doi.org/10.5937/fmet1801117P

[11] H. Ozbasaran, solveTruss v1.0: Static, global buckling and frequency analysis of $2 \mathrm{D}$ and $3 \mathrm{D}$ trusses with Mathematica. SoftwareX, 6, 2017: 135-140.

https://doi.org/10.1016/j.softx.2017.05.004

[12] N. Petrovic, N. Kostic, N. Marjanovic, Discrete Variable Truss Structural Optimization Using Buckling Dynamic Constraints. Machine Design, 10 (2), 2018: 51-56.

https://doi.org/10.24867/MD.10.2018.2.51-56

[13] N. Petrović, N. Kostić, N. Marjanović, V. Marjanović, Influence of Using Discrete CrossSection Variables for All Types of Truss Structural Optimization with Dynamic Constraints for Buckling. Applied Engineering Letters, 3 (2), 2018: 78-83.

https://doi.org/10.18485/aeletters.2018.3.2.5

[14] M. Reda, T. Sharaf, A. ElSabbagh, M. ElGhandour, Behavior and design for component and system of cold-formed steel roof trusses. Thin-Walled Structures, 135 2019: 21-32. 
https://doi.org/10.1016/j.tws.2018.10.038

[15] J.L. Dawe, Y. Liu, J.Y. Li, Strength and behaviour of cold-formed steel offset trusses. Journal of Constructional Steel

Research, 66 (4), 2010: 556-565.

https://doi.org/10.1016/j.jcsr.2009.10.015 